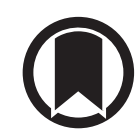

CrossMark

\section{Increasing bronchiectasis prevalence in Germany, 2009-2017: a population-based cohort study}

\author{
To the Editor:
}

Bronchiectasis is a chronic airway disease with often disabling symptoms, which is associated with excess mortality and a substantial economic burden for healthcare systems [1]. Although considered to be one of the most neglected diseases in respiratory medicine [2], bronchiectasis is apparently more common than previously thought $[3,4]$. While trends regarding its epidemiology have been published for the UK and the USA, with marked increases in prevalence rates reported [5-7], such studies are missing for most countries including Germany. However, these basic epidemiological data are needed in order to inform healthcare authorities and policy makers regarding resource allocation and requirements planning. Thus, the objective of the present study was to provide insights into the trends of bronchiectasis prevalence in Germany.

Our study was based on the externally validated InGef research database [8], which contains anonymised patient-level claims data from approximately 7 million insured mandatory or voluntary members of the German public health scheme, including data from several German statutory health insurance (SHI) companies, regardless of their social security status (dependently employed, self-employed or unemployed subjects, dependently insured spouses and/or children, pensioners, subjects on disability or sick-leave, etc.). Briefly, between 2009 and 2017, approximately 70 of 81 million population (86\%) were members of the public health scheme, while the rest had private health coverage [9]. Samples of approximately four million insured persons per year were randomly selected from the entire InGef database. These samples were representative of the German population regarding age and sex [9]. Finally, all patients with complete data and continuous SHI coverage in the respective periods served as the study population, including on average $96 \%$ (range $90-100 \%$ ) of subjects from the initial random samples. This resulted in representative $\sim 5 \%$ samples of the German population covered by SHI for each year between 2009 and 2017. Data were extracted using the ICD-10 diagnosis code J47 (acquired bronchiectasis) as primary or secondary hospital discharge or verified outpatient diagnosis. Subsequently, data were analysed according to age, sex, the sector of healthcare provision (hospital or outpatient care), and chronic obstructive pulmonary disease (COPD)/emphysema and asthma as concomitant diagnoses (ICD-10 codes J43-44 and J45-46, respectively). Subjects with cystic fibrosis (ICD-10 code E84) were excluded from analysis. Official census data were obtained from the German Federal Statistical Office [9]. Poisson log-linear regression analysis was used to assess the significance of prevalence trend (IBM SPSS Statistics, version 25; IBM Corp., New York, NY, USA). Prevalence rates and 95\% confidence intervals were calculated using OpenEpi version 3.03a [10]. In order to extrapolate the total number of subjects with bronchiectasis in the German population per year, the number of bronchiectasis case-patients was divided by the total number of subjects in the study samples for each year and multiplied with the total number of the German population according to official census data [9]. Then, the total increase of extrapolated bronchiectasis patients was calculated as percent increase from baseline (2009) compared with 2017. The average annual percentage increase was calculated by dividing the total increase by the number of subsequent time intervals $(n=8)$. Ethical approval was not required, as the present study analysed anonymous routine SHI claim data.

Between 2009 and 2017, the annual overall prevalence of bronchiectasis increased significantly from 52.5 (95\% CI 50.3-54.9) to 94.8 (95\% CI 91.7-97.9) per 100000 population $(\mathrm{p}<0.001)$, representing an average

@ERSpublications

Bronchiectasis prevalence in Germany increased on average by 10\% per year between 2009 and 2017 http://bit.ly/2P4KcyW

Cite this article as: Ringshausen FC, Rademacher J, Pink I, et al. Increasing bronchiectasis prevalence in Germany, 2009-2017: a population-based cohort study. Eur Respir J 2019; 54: 1900499 [https://doi.org/ 10.1183/13993003.00499-2019]. 
annual increase of $10 \%$. Accordingly, the total number of case-patients in the study sample as well as the extrapolated number of patients with bronchiectasis in the general population increased steadily from 1981 to 3664 and from 42977 to 78450 , respectively. In this period, the mean age of case-patients continuously increased from 64.2 (95\% CI 63.5-64.9) to 67.7 (95\% CI 67.2-68.2) years in agreement with the increasing mean age in the general population (data not shown), but did not differ between men and women. The sex ratio was comparatively balanced throughout, with a slight female predominance (range 51.0\% (95\% CI 48.7-53.3; in 2017) to 53.5\% (95\% CI 50.4-56.8; in 2010)). Figure 1 shows the annual prevalence of bronchiectasis stratified by age and sex from 2009 to 2017. Annual overall prevalence rates were generally slightly higher among females. While we observed a marked overall increase in prevalence among subjects aged $\geqslant 60$ years, females aged $\geqslant 80$ years experienced the most pronounced increase of $197 \%$ (figure 1 ). The highest age- and sex-specific prevalence was observed in the age group of 70-79 years in 2017 (332.6 and 313.1 per 100000 population among males and females, respectively; figure 1). The vast majority of case-patients were managed in outpatient care (range 91.4\% (95\% CI 88.0-94.8; in 2015) to 93.5\% (95\% CI 89.3-97.9; in 2009)), while the proportion of hospitalised subjects ranged from $12.6 \%$ (95\% CI 11.1 14.3 ; in 2009 ) to $16.1 \%$ (95\% CI 14.7-17.7; in 2014). Notably, about one half of hospitalised case-patients were not diagnosed with bronchiectasis in outpatient care in the respective years (range 47.6\% (95\% CI $40.0-56.2$; in 2010$)$ to $57.1 \%$ (95\% CI $49.0-66.2$; in 2011)), potentially indicating a lack of specific follow-up. As expected, COPD/emphysema and asthma were among the most prevalent concomitant diagnoses (range 56.9\% (95\% CI 53.7-60.4, in 2009) to 61.8\% (95\% CI 59.1-64.6; in 2016)) and $33.1 \%$ (95\% CI 30.8-35.6, in 2011) to $37.5 \%$ (35.4-39.7; in 2016), respectively).

The present population-based study found an increasing overall prevalence of bronchiectasis in Germany between 2009 and 2017, with an average annual increase of approximately 10\%, which was most pronounced among the elderly. The finding of an increasing prevalence is in line with our previous study, which showed a steady annual increase of bronchiectasis-associated hospitalisations of 3\% between 2005 and 2011 [11]. In addition, our prevalence and growth rates are comparable to those determined by a population-based study in the US, which demonstrated an overall period prevalence of bronchiectasis of 139 per 100000 US adults $\geqslant 18$ years between 2009 and 2013, corresponding to an average annual increase of $8 \%$ since 2001 (prevalence in 2001: 52 per 100000 adults) [12, 13]. However, our results are well below the rates recently identified by two population-based studies from the US and the UK [6, 7]. HenKLE et al. [7] found an average prevalence of 701 per 100000 among Medicare insured subjects aged $\geqslant 65$ years with pulmonologist-diagnosed bronchiectasis during 2012-2014, while in a primary care-based study from the UK, QUINT et al. [6] described a remarkable increase from 301 to 486 per 100000 population among men and from 351 to 566 per 100000 population among women during 2004-2013. Nevertheless, in the study by HeNkLe et al. [7] the proportion of subjects with concomitantly diagnosed COPD and asthma was $51 \%$ and $28 \%$, which is in good agreement with our findings.

Our study has several limitations. It should be mentioned that the ICD-10 code for bronchiectasis ( J47), which we used to identify case-patients, has been developed primarily for reimbursement purposes, has unknown sensitivity and specificity for the diagnosis of bronchiectasis in our study population and does not require confirmation by chest computed tomography. However, our data likely underestimate the true prevalence of bronchiectasis, as its diagnosis usually does not trigger specific prescriptions due to the lack of approved pharmacological treatments and restrictions of prescribing chest physiotherapy in Germany. While our study includes representative $5 \%$ samples of the German population with public health scheme
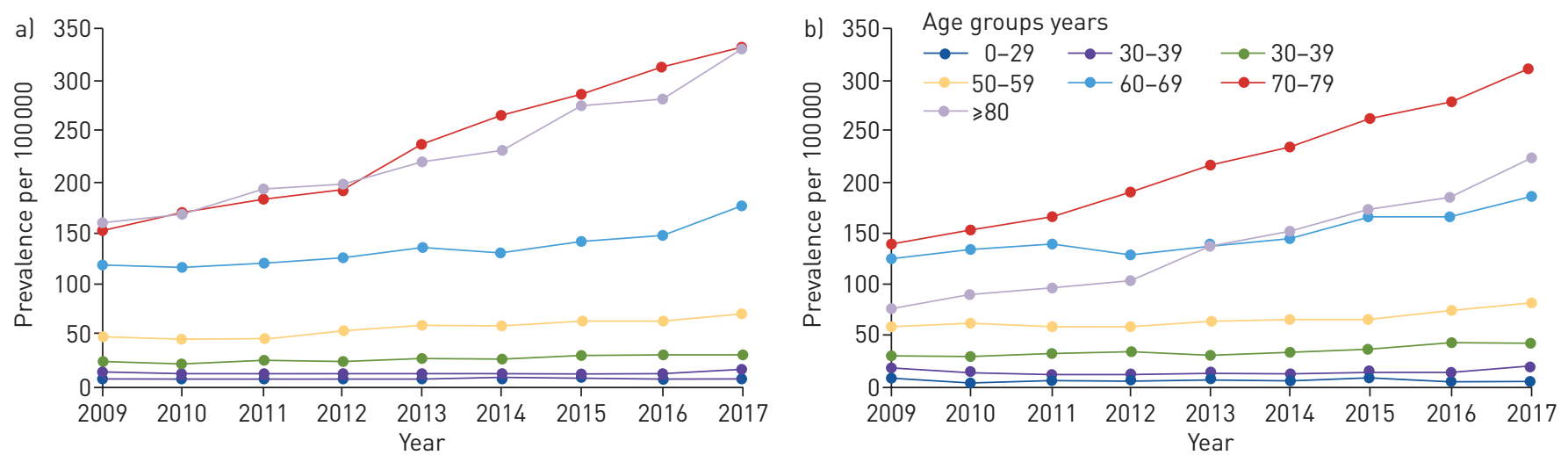

FIGURE 1 Prevalence of bronchiectasis in Germany from 2009 to 2017, stratified by age group, in a) men and b) women. 
and can thus be regarded as robust, our results may not apply to privately insured individuals and other healthcare systems. In addition, subjects from East Germany are under-represented in the InGef database. Although, the study population is representative of the German population with regard to age and sex and thus regional influences on the prevalence of bronchiectasis appear unlikely, we cannot fully exclude bias to a minor degree here. Discrepancies between the present work and other recent epidemiological studies concerning prevalence and the proportion of female case-patients, which were up to $67 \%$ in US studies [7, 12], may point towards differences in underlying data sources (SHI claim data, primary or secondary care data), healthcare systems $[1,6,7,12]$ and an age- and sex-specific utilisation of health services in Germany [9, 11]. Although the definite reasons for the observed increase in annual prevalence rates remain unknown, they may be explained, at least in part, by the demographic change of society, the lower threshold for using chest computed tomography and, very recently, growing awareness driven by collaborative research initiatives $[14,15]$.

Our study highlights the relevance of bronchiectasis, in particular when bearing in mind the associated mortality and economic burden [1]. Overall, $\geqslant 90 \%$ of bronchiectasis patients were treated in the outpatient setting, where budgeting and the risk of refund claims for off-label prescriptions hamper appropriate patient management in Germany. The high proportion of subjects with COPD and asthma in our study reflects a considerable overlap in clinical presentation [16]. In this regard, our findings call for continued efforts for earlier and more effective interventions in order to prevent the development of chronic airflow limitation in bronchiectasis as well as the progression to bronchiectasis in obstructive lung diseases.

In conclusion, our study provides evidence that the patient group with bronchiectasis has been growing at a rate of 10\% per year between 2009 and 2017, thus emphasising its relevance for the German healthcare system as well as the urgent need for specialised multidisciplinary teams, in particular in the outpatient setting.

Felix C. Ringshausen $\oplus^{1,2}$, Jessica Rademacher ${ }^{1,2}$, Isabell Pink ${ }^{1}$, Andrés de Roux ${ }^{3}$, Lennart Hickstein ${ }^{4}$, Tina Ploner ${ }^{4}$, Tobias Welte ${ }^{1,2}$ and Roland Diel ${ }^{5,6,7}$

${ }^{1}$ Dept of Respiratory Medicine, Hannover Medical School, Hannover, Germany. ${ }^{2}$ Biomedical Research in End-stage and Obstructive Lung Disease Hannover (BREATH), German Center for Lung Research (DZL), Hannover, Germany. ${ }^{3}$ Pneumologische Praxis am Schloss Charlottenburg, Berlin, Germany. ${ }^{4}$ InGef - Institute for Applied Health Research Berlin GmbH, Berlin, Germany. ${ }^{5}$ Institute for Epidemiology, University Medical Center Schleswig-Holstein, Kiel, Germany. ${ }^{6}$ LungenClinic Grosshansdorf, Airway Research Center North (ARCN), German Center for Lung Research (DZL), Grosshansdorf, Germany. ${ }^{7}$ Institution for Statutory Accident Insurance and Prevention in the Health and Welfare Services (BGW), Hamburg, Germany.

Correspondence: Felix C. Ringshausen, Dept of Respiratory Medicine, Hannover Medical School, Carl-Neuberg-Strasse 1, 30625 Hannover, Germany. E-mail: Ringshausen.Felix@mh-hannover.de

Received: 10 Mar 2019 | Accepted after revision: 13 Aug 2019

Acknowledgement: We thank the team of Biomedical Research in End-stage and Obstructive Lung Disease Hannover (BREATH), Annegret Zurawski, Inga Kwapniewska and Anne Hösel for their great support of bronchiectasis research within the German Center for Lung Research (DZL).

Conflict of interest: F.C. Ringshausen reports grants, personal fees for consultancy and lectures, and support for educational activities from Bayer HealthCare and Grifols Germany, grants, personal fees for consultancy and lectures, support for educational activities and clinical trial participation from Insmed Germany and Novartis, personal fees for consultancy and lectures from AstraZeneca, personal fees for lectures, support for educational activities and clinical trial participation from from Chiesi and Boehringer Ingelheim, grants and support for educational activities from InfectoPharm, support for clinical trial participation from Vertex, Parion, Celtaxsys, Corbus and Algipharma, support for educational activities and clinical trial participation from from GSK, grants from Polyphor and Baslilea, personal fees for consultancy and support for clinical trial participation from Zambon, outside the submitted work. J. Rademacher reports grants and personal fees from Bayer Health Care, Insmed and Grifols, personal fees from MSD Sharp \& Dohme, AstraZeneca and Chiesi, outside the submitted work. I. Pink reports grants from Infectopharm, Bayer, Insmed and Grifols, during the conduct of the study; personal fees and non-financial support from Berlin Chemie, Boehringer Ingelheim and Chiesi, outside the submitted work. A. de Roux has nothing to disclose. L. Hickstein has nothing to disclose. T. Ploner has nothing to disclose. T. Welte reports grants and personal fees for lectures from Grifols, Insmed and Novartis, outside the submitted work. R. Diel reports grants and personal fees from Bayer Vital, personal fees from Insmed Inc., outside the submitted work.

\section{References}

1 Diel R, Chalmers JD, Rabe KF, et al. Economic burden of bronchiectasis in Germany. Eur Respir J 2019; 53: 1802033.

2 Bronchiectasis. In: Gibson J, Loddenkemper R, Sibille Y, et al., eds. The European Lung White Book: Respiratory Health and Disease in Europe. Lausanne, European Respiratory Society, 2013; pp. 176-183.

3 Chalmers JD. New insights into the epidemiology of bronchiectasis. Chest 2018; 154: 1272-1273. 
4 Ringshausen FC, de Roux A, Diel R, et al. Bronchiectasis in Germany: a population-based estimation of disease prevalence. Eur Respir J 2015; 46: 1805-1807.

5 Seitz AE, Olivier KN, Adjemian J, et al. Trends in bronchiectasis among Medicare beneficiaries in the United States, 2000-2007. Chest 2012; 142: 432-439.

6 Quint JK, Millett ER, Joshi M, et al. Changes in the incidence, prevalence and mortality of bronchiectasis in the UK from 2004 to 2013: a population-based cohort study. Eur Respir J 2016; 47: 186-193.

7 Henkle E, Chan B, Curtis JR, et al. Characteristics and health-care utilization history of patients with bronchiectasis in US Medicare enrollees with prescription drug plans, 2006 to 2014. Chest 2018; 154: 1311-1320.

8 Andersohn F, Walker J. Characteristics and external validity of the German Health Risk Institute (HRI) Database. Pharmacoepidemiol Drug Saf 2016; 25: 106-109.

9 German Federal Statistical Office. Facts \& Figures - State \& Society - Federal Statistical Office (Destatis). 2019. www.destatis.de/EN/FactsFigures/SocietyState/SocietyState.html. Date last updated: February 27, 2019. Date last accessed: February 27, 2019.

10 Dean AG, Sullivan KM, Soe MM. OpenEpi: Open Source Epidemiologic Statistics for Public Health. http:// openepi.com/Menu/OE_Menu.htm. Date last updated: Apr 6, 2013. Date last accessed: Mar 1, 2019.

11 Ringshausen FC, de Roux A, Pletz MW, et al. Bronchiectasis-associated hospitalizations in Germany, 2005-2011: a population-based study of disease burden and trends. PLoS One 2013; 8: e71109.

12 Weycker D, Hansen GL, Seifer FD. Prevalence and incidence of noncystic fibrosis bronchiectasis among US adults in 2013. Chron Respir Dis 2017; 14: 377-384.

13 Weycker D, Edelsberg J, Oster G, et al. Prevalence and economic burden of bronchiectasis. Clin Pulm Med 2005; 12: 205-209.

14 Chalmers JD, Aliberti S, Polverino E, et al. The EMBARC European Bronchiectasis Registry: protocol for an international observational study. ERJ Open Res 2016; 2: 00081-02015.

15 Winter DH, Manzini M, Salge JM, et al. Aging of the lungs in asymptomatic lifelong nonsmokers: findings on HRCT. Lung 2015; 193: 283-290.

16 Polverino E, Dimakou K, Hurst J, et al. The overlap between bronchiectasis and chronic airway diseases: state of the art and future directions. Eur Respir J 2018; 52: 1800328. 